\title{
Some generalized inequalities of Hermite-Hadamard type for strongly $s$-convex functions
}

\author{
Yusuf Erdem ${ }^{1}$, Hasan Ogunmez ${ }^{1}$ and Huseyin Budak ${ }^{2}$ \\ ${ }^{1}$ Department of Mathematics, Faculty of Science and Arts, Afyon Kocatepe University, Afyonkarahisar, Turkey \\ ${ }^{2}$ Department of Mathematics, Faculty of Science and Arts, Duzce University, Duzce, Turkey
}

Received: 18 November 2016, Accepted: 7 December 2016

Published online: 17 August 2017.

\begin{abstract}
In this paper, some new generalized results related to the left-hand and the right-hand of the Hermite-Hadamard inequalities for the class of functions whose derivatives are strongly s-convex functions in the second sense are established. Some previous results are also recaptured as a special case.
\end{abstract}

Keywords: Hadamard type inequalities, Hölder inequality, strongly $s$-convex functions.

\section{Introduction}

In this section, we firstly list several definitions and some known results.

Definition 1. A function $f: I \rightarrow \mathbb{R}, \varnothing \neq I \subset \mathbb{R}$, is said to be convex on I if the inequality

$$
f(t x+(1-t) y) \leq t f(x)+(1-t) f(y)
$$

holds for all $x . y \in I$ and $t \in[0,1]$.

Many inequalities have been established for convex functions but the most famous inequality is the Hermite-Hadamards inequality, due to its rich geometrical significance and applications([4], [12, p.137]). These inequalities state that if $f: I \subseteq R \rightarrow R$ is a convex function on the interval $I$ of real numbers and $a, b \in I$ with $a<b$, then

$$
f\left(\frac{a+b}{2}\right) \leq \frac{1}{b-a} \int_{a}^{b} f(x) d x \leq \frac{f(a)+f(b)}{2}
$$

Both inequalities hold in the reversed direction if $f$ is concave. We note that Hadamards inequality may be regarded as a refinement of the concept of convexity and it follows easily from Jensens inequality. Hadamards inequality for convex functions has received renewed attention in recent years and a remarkable variety of refinements and generalizations have been found (see, for example, [2],[5],[11],[16],[18]) and the references cited therein.

In [6], Hudzik and Maligranda considered, among others, the class of functions which are s- convex in the second sense. This class is defined in the following way: a function $f:[0, \infty) \rightarrow \mathbb{R}$ is said to be s-convex in the second sense if

$$
f(t x+(1-t) y) \leq t^{s} f(x)+(1-t)^{s} f(y)
$$


holds for $x, y \in[0, \infty), t \in[0,1]$ and for some fixed $s \in[0,1]$. This class of s-convex functions in the second sense is usually by $K_{S}^{2}$.

It can be easily see that for $s=1 \mathrm{~s}$-convexity reduces to the ordinary convexity of functions defined on $[0, \infty)$.

Definition 2. [13] A function $f: I \rightarrow \mathbb{R}$ is called strongly s-convex with modulus $c$ if

$$
f(t x+(1-t) y) \leq t^{s} f(x)+(1-t)^{s} f(y)-c t(1-t)(b-a)^{2} .
$$

In [1], Angulo et al. proved the following Hermite-Hadamard type inequality for strongly $h$-convex function.

Theorem 1. Let $h:(0,1) \rightarrow(0, \infty)$ be a given function. If a function $f: I \subset \mathbb{R} \rightarrow \mathbb{R}$ is Lebesgue integrable and strongly $h$-convex with modulus $c>0$, then

$$
\frac{1}{h\left(\frac{1}{2}\right)}\left[f\left(\frac{a+b}{2}\right)+\frac{c}{12}(b-a)^{2}\right] \leq \frac{1}{b-a} \int_{a}^{b} f(x) d x \leq(f(a)+f(b)) \int_{0}^{1} h(t) d t-\frac{c}{6}(b-a)^{2}
$$

for all $a, b \in I, a<b$.

Corollary 1. Suppose that $f:[0, \infty) \rightarrow \mathbb{R}$ is a strongly $s$-convex function in the second sense with modulus $c>0$, where $s \in(0,1)$ (i.e $h(t)=t^{s}$ in (2)), then following inequalities hold;

$$
2^{s-1}\left[f\left(\frac{a+b}{2}\right)+\frac{c}{12}(b-a)^{2}\right] \leq \frac{1}{b-a} \int_{a}^{b} f(x) d x \leq \frac{f(a)+f(b)}{s+1}-\frac{c}{6}(b-a)^{2} .
$$

For more information and recent developments on inequalities for strongly convex function, please refer to ([1],[3],[8],[9],[10],[15],[17],[19],[20]).

To prove our main results, we consider the following Lemmas given by Sarikaya et al. in [14] and Kiriş and Sarikaya in [7], respectively:

Lemma 1. Let $f: I \rightarrow \mathbb{R}$ be a differantiable mapping on $I^{0}$ where $a, b \in I$ with $a<b$. If $f^{\prime} \in L[a, b]$, then we have

$$
\int_{a}^{b} p(x) f^{\prime}(x) d x=(m-n) f\left(\frac{a+b}{2}\right)+(b-m) f(b)+(n-a) f(a)-\int_{a}^{b} f(x) d x
$$

where

$$
p(x)=\left\{\begin{array}{l}
x-n, x \in\left[a, \frac{a+b}{2}\right] \\
x-m, x \in\left(\frac{a+b}{2}, b\right]
\end{array}\right\}
$$

for $n \in\left[a, \frac{a+b}{2}\right]$ and $m \in\left[\frac{a+b}{2}, b\right]$.

Lemma 2. Let $f: I \rightarrow \mathbb{R}$ be a differantiable mapping on $I^{0}$ where $a, b \in I$ with $a<b$. If $f^{\prime} \in L[a, b]$, then we have

$$
\frac{n}{b-a} \int_{a}^{\frac{a+b}{2}} f(x) d x+\frac{m}{b-a} \int_{\frac{a+b}{2}}^{b} f(x) d x-\frac{n+m}{2} f\left(\frac{a+b}{2}\right)=(b-a) w f
$$


where $w f=\left[\int_{0}^{\frac{1}{2}} m t f^{\prime}((t a)+(1-t) b) d t+\int_{\frac{1}{2}}^{1} n(1-t) f^{\prime}((t a)+(1-t) b) d t\right], n, m>0$

The aim of the paper is to establish some new generalized Hermite-Hadamard inequalities for function whose derivatives absolute values are strongly $s-$ convex.

\section{Main results}

Firstly, we will give some calculated integrals which used our main results:

$$
\begin{aligned}
& \int_{a}^{\frac{a+b}{2}}|x-n|(b-x)^{s} d x=\frac{2(b-n)^{s+2}}{(s+1)(s+2)}-\frac{(b-n)(b-a)^{s+1}\left[2^{s+1}+1\right]}{2^{s+1}(s+1)}+\frac{(b-a)^{s+2}\left[2^{s+2}+1\right]}{2^{s+2}(s+2)}, \\
& \int_{\frac{a+b}{2}}^{b}|x-m|(b-x)^{s}=\frac{2(b-m)^{s+2}}{(s+1)(s+2)}+\frac{(b-a)^{s+2}}{2^{s+2}(s+2)}-\frac{(b-m)(b-a)^{s+1}}{2^{s+1}(s+1)}, \\
& \int_{a}^{\frac{a+b}{2}}|x-n|(x-a)^{s} d x=\frac{2(n-a)^{s+2}}{(s+1)(s+2)}+\frac{(b-a)^{s+2}}{2^{s+2}(s+2)}-\frac{(n-a)(b-a)^{s+1}}{2^{s+1}(s+1)}, \\
& \int_{\frac{a+b}{2}}^{b}|x-m|(x-a)^{s} d x=\frac{2(m-a)^{s+2}}{(s+1)(s+2)}-\frac{(m-a)(b-a)^{s+1}\left[2^{s+1}+1\right]}{2^{s+1}(s+1)}+\frac{(b-a)^{s+2}\left[2^{s+2}+1\right]}{2^{s+2}(s+2)}, \\
& \int_{a}^{\frac{a+b}{2}}|x-n|(b-x)(x-a) d x=\frac{(b-a)(n-a)^{3}}{3}-\frac{(n-a)^{4}}{6}+\frac{5(b-a)^{4}}{192}-\frac{(n-a)(b-a)^{3}}{12}
\end{aligned}
$$

and

$$
\int_{\frac{a+b}{2}}^{b}|x-m|(b-x)(x-a) d x=\frac{(b-a)(b-m)^{3}}{3}-\frac{(b-m)^{4}}{6}+\frac{5(b-a)^{4}}{192}-\frac{(b-m)(b-a)^{3}}{12} .
$$

Theorem 2. Let $f: I \rightarrow \mathbb{R}$ be a differantiable mapping on $I^{0}$ where $a, b \in I$ with $a<b$. If $\left|f^{\prime}\right|$ is strongly s-convex on $[a, b]$, for some $s \in(0,1]$ with modulus $c>0$, then following inequality holds :

$$
\begin{aligned}
& \left|(m-n) f\left(\frac{a+b}{2}\right)+(b-m) f(b)+(n-a) f(a)-\int_{a}^{b} f(x) d x\right| \leq \frac{\left|f^{\prime}(a)\right|}{(b-a)^{s}}\left[\frac{2\left[(b-m)^{s+2}+(b-n)^{s+2}\right]}{(s+1)(s+2)}\right. \\
& \left.-\frac{(2 b-n-m)(b-a)^{s+1}}{2^{s+1}(s+1)}-\frac{(b-n)(b-a)^{s+1}}{(s+1)}+\frac{(b-a)^{s+2}\left[2^{s+1}+1\right]}{2^{s+1}(s+2)}\right]+\frac{\left|f^{\prime}(b)\right|}{(b-a)^{s}}\left[\frac{2\left[(b-m)^{s+2}+(b-n)^{s+2}\right]}{(s+1)(s+2)}\right. \\
& \left.-\frac{(m+n-2 a)(b-a)^{s+1}}{2^{s+1}(s+1)}-\frac{(m-a)(b-a)^{s+1}}{(s+1)}+\frac{(b-a)^{s+2}\left[2^{s+1}+1\right]}{2^{s+1}(s+2)}\right] \\
& -c\left[(b-a) \frac{(n-a)^{3}+(b-m)^{3}}{3}-\frac{(n-a)^{4}+(b-m)^{4}}{6}+\frac{5(b-a)^{4}}{96}-\frac{((b-a)-(m-n))(b-a)^{3}}{12}\right]
\end{aligned}
$$

for $n \in\left[a, \frac{a+b}{2}\right]$ and $m \in\left[\frac{a+b}{2}, b\right]$. 
Proof. Taking modulus in Lemma 1 and using the strongly $s$-convexity of $\left|f^{\prime}\right|$, we have

$$
\begin{aligned}
& \left|(m-n) f\left(\frac{a+b}{2}\right)+(b-m) f(b)+(n-a) f(a)-\int_{a}^{b} f(x) d x\right| \\
& \leq \int_{a}^{\frac{a+b}{2}}|x-n|\left|f^{\prime}(x)\right| d x+\int_{\frac{a+b}{2}}^{b}|x-m|\left|f^{\prime}(x)\right| d x \\
& =\int_{a}^{\frac{a+b}{2}}|x-n| f\left(\frac{b-x}{b-a} a+\frac{x-a}{b-b} b\right) d x+\int_{\frac{a+b}{2}}^{b}|x-m| f\left(\frac{b-x}{b-a} a+\frac{x-a}{b-b} b\right) d x \\
& \leq \int_{a}^{\frac{a+b}{2}}|x-n|\left[\left(\frac{b-x}{b-a}\right)^{s}\left|f^{\prime}(a)\right|+\left(\frac{x-a}{b-a}\right)^{s}\left|f^{\prime}(b)\right|-c(b-x)(x-a)\right] d x \\
& +\int_{\frac{a+b}{2}}^{b}|x-m|\left[\left(\frac{b-x}{b-a}\right)^{s}\left|f^{\prime}(a)\right|+\left(\frac{x-a}{b-a}\right)^{s}\left|f^{\prime}(b)\right|-c(b-x)(x-a)\right] d x \\
& =\frac{\left|f^{\prime}(a)\right|}{(b-a)^{s}}\left[\int_{a}^{\frac{a+b}{2}}|x-n|(b-x)^{s} d x+\int_{\frac{a+b}{2}}^{b}|x-m|(b-x)^{s} d x\right] \\
& +\frac{\left|f^{\prime}(b)\right|}{(b-a)^{s}}\left[\int_{a}^{\frac{a+b}{2}}|x-n|(x-a)^{s} d x+\int_{\frac{a+b}{2}}^{b}|x-m|(x-a)^{s} d x\right] \\
& -c\left[\int_{a}^{\frac{a+b}{2}}|x-n|(b-x)(x-a) d x+\int_{\frac{a+b}{2}}^{b}|x-m|(b-x)(x-a) d x\right] \text {. }
\end{aligned}
$$

If we substitute the equalities (6)-(11) in (13), then we obtain required result (12).

Remark. If we choose $m=b, n=a$ in Theorem 2, then we have

$$
\left|f\left(\frac{a+b}{2}\right)-\frac{1}{b-a} \int_{a}^{b} f(x) d x\right| \leq(b-a) \frac{2^{s+1}-1}{2^{s}(s+1)(s+2)}\left[\frac{\left|f^{\prime}(a)\right|+\left|f^{\prime}(b)\right|}{2}\right]-\frac{5 c}{96}(b-a)^{3}
$$

Remark. If we choose $m=n=\frac{a+b}{2}$ in Theorem 2, then we have

$$
\left|\frac{f(a)+f(b)}{2}-\frac{1}{b-a} \int_{a}^{b} f(x) d x\right| \leq(b-a) \frac{s 2^{s}+1}{2^{s}(s+1)(s+2)}\left[\frac{\left|f^{\prime}(a)\right|+\left|f^{\prime}(b)\right|}{2}\right]-\frac{c}{32}(b-a)
$$


Remark. If we choose $m=\frac{a+5 b}{6}, n=\frac{5 a+b}{6}$ in Theorem 2, then we have

$$
\begin{aligned}
\left|\frac{1}{6}\left[f(a)+4 f\left(\frac{a+b}{2}\right)+f(b)\right]-\frac{1}{b-a} \int_{a}^{b} f(x) d x\right| \leq & (b-a) \frac{2 \times 5^{s+2}-(4-s) 6^{s+1}-2 \times 3^{s+12}+2}{6^{s+2}(s+1)(s+2)}\left[\left|f^{\prime}(a)\right|+\left|f^{\prime}(b)\right|\right] \\
& -\frac{67}{2 \times 6^{4}} c(b-a)^{3}
\end{aligned}
$$

For $c=0$ it reduces to the Hermite-Hadamard-type inequalities for $s$-convex functions proved by Sarikaya et al. in [21].

Theorem 3. Let $f: I \rightarrow \mathbb{R}$ be a differantiable mapping on $I^{0}$ where $a, b \in I$ with $a<b$. If $\left|f^{\prime}\right|^{q}$ is strongly s-convex on $[a, b]$ for some $s \in(0,1]$ with modulus $c>0$, then following inequality holds.

$$
\begin{aligned}
& \left|(m-n) f\left(\frac{a+b}{2}\right)+(b-m) f(b)+(n-a) f(a)-\int_{a}^{b} f(x) d x\right| \leq \frac{(b-a)^{\frac{1}{q}}}{(p+1)^{\frac{1}{p}}}\left\{\left[(n-a)^{p+1}+\left(\frac{a+b}{2}-n\right)^{p+1}\right]^{\frac{1}{p}}\right. \\
& \times\left(\frac{1}{2^{s+1}(s+1)}\left[\left[2^{s+1}-1\right]\left|f^{\prime}(a)\right|^{q}+\left|f^{\prime}(b)\right|^{q}\right]-c \frac{(b-a)^{2}}{12}\right)^{\frac{1}{q}}+\left[(b-m)^{p+1}+\left(m-\frac{a+b}{2}\right)^{p+1}\right]^{\frac{1}{p}} \\
& \left.\times\left(\frac{1}{2^{s+1}(s+1)}\left[\left|f^{\prime}(a)\right|^{q}+\left[2^{s+1}-1\right]\left|f^{\prime}(b)\right|^{q}\right]-c \frac{(b-a)^{2}}{12}\right)^{\frac{1}{q}}\right\}
\end{aligned}
$$

for $n \in\left[a, \frac{a+b}{2}\right]$ and $m \in\left[\frac{a+b}{2}, b\right]$ where $p, q>1, \frac{1}{p}+\frac{1}{q}=1$.

Proof. From Lemma 1 and by using the Hölder inequality, then we have

$$
\begin{aligned}
& \left|(m-n) f\left(\frac{a+b}{2}\right)+(b-m) f(b)+(n-a) f(a)-\int_{a}^{b} f(x) d x\right| \\
& =\int_{a}^{\frac{a+b}{2}}|x-n|\left|f^{\prime}(x)\right| d x+\int_{\frac{a+b}{2}}^{b}|x-m|\left|f^{\prime}(x)\right| d x \\
& \leq\left(\int_{a}^{\frac{a+b}{2}}|x-n|^{p} d x\right)^{\frac{1}{p}}\left(\int_{a}^{\frac{a+b}{2}}\left|f^{\prime}(x)\right|^{q} d x\right)^{\frac{1}{q}}+\left(\int_{\frac{a+b}{2}}^{b}|x-m|^{p} d x\right)^{\frac{1}{p}}\left(\int_{\frac{a+b}{2}}^{b}\left|f^{\prime}(x)\right|^{q} d x\right)^{\frac{1}{q}} \\
& =\frac{1}{(p+1)^{\frac{1}{p}}}\left[(n-a)^{p+1}+\left(\frac{a+b}{2}-n\right)^{\frac{1}{q}}\right]^{\frac{1}{p}}\left(\int_{a}^{\frac{a+b}{2}}\left|f^{\prime}(x)\right|^{q} d x\right)^{\frac{1}{q}} \\
& +\frac{1}{(p+1)^{\frac{1}{p}}}\left[(b-m)^{p+1}+\left(m-\frac{a+b}{2}\right)^{p+1}\right]^{\frac{1}{p}}\left(\int_{\frac{a+b}{2}}^{b}\left|f^{\prime}(x)\right|^{q} d x\right)^{\frac{1}{q}}
\end{aligned}
$$


Using the strongly s-convexity of $\left|f^{\prime}\right|^{q}$, we have

$$
\begin{aligned}
& \left|(m-n) f\left(\frac{a+b}{2}\right)+(b-m) f(b)+(n-a) f(a)-\int_{a}^{b} f(x) d x\right| \\
& \leq \frac{1}{(p+1)^{\frac{1}{p}}}\left\{\left[(n-a)^{p+1}+\left(\frac{a+b}{2}-n\right)^{p+1}\right]^{\frac{1}{p}}\right. \\
& \times\left(\int_{a}^{\frac{a+b}{2}}\left[\left(\frac{b-x}{b-a}\right)^{s}\left|f^{\prime}(a)\right|^{q}+\left(\frac{x-a}{b-a}\right)^{s}\left|f^{\prime}(b)\right|^{q}-c(b-x)(x-a)\right] d x\right)^{\frac{1}{q}} \\
& +\left[(b-m)^{p+1}+\left(m-\frac{a+b}{2}\right)^{p+1}\right]^{\frac{1}{p}} \\
& \times\left(\int_{\frac{a+b}{2}}^{b}\left[\left(\frac{b-x}{b-a}\right)^{s}\left|f^{\prime}(a)\right|^{q}+\left(\frac{x-a}{b-a}\right)^{s}\left|f^{\prime}(b)\right|^{q}-c(b-x)(x-a)\right] d x\right)^{\frac{1}{q}}
\end{aligned} .
$$

By simple computation, we have

$$
\begin{aligned}
& \int_{a}^{\frac{a+b}{2}}(b-x)^{s} d x=\int_{\frac{a+b}{2}}^{b}(x-a)^{s} d x=\frac{(b-a)^{s+1}\left[2^{s+1}-1\right]}{2^{s+1}(s+1)} \\
& \int_{a}^{\frac{a+b}{2}}(x-a)^{s} d x=\int_{\frac{a+b}{2}}^{b}(b-x)^{s} d x=\frac{(b-a)^{s+1}}{2^{s+1}(s+1)} \\
& \int_{a}^{\frac{a+b}{2}}(b-x)(x-a) d x=\int_{\frac{a+b}{2}}^{b}(b-x)(x-a) d x=\frac{(b-a)^{3}}{12} .
\end{aligned}
$$

If we substitute the equalities (17)-(19) in (16), then we obtain result (14).

Corollary 2. If we choose $m=b$ and $n=a$ in Theorem 3, then we have

$$
\begin{aligned}
\left|f\left(\frac{a+b}{2}\right)-\int_{a}^{b} f(x) d x\right| \leq & \frac{b-a}{4}\left(\frac{1}{p+1}\right)^{\frac{1}{p}}\left\{\left(\frac{\left[2^{s+1}-1\right]\left|f^{\prime}(a)\right|^{q}+\left|f^{\prime}(b)\right|^{q}}{2^{s}(s+1)}-c \frac{(b-a)^{2}}{6}\right)^{\frac{1}{q}}\right. \\
& \left.+\left(\frac{\left|f^{\prime}(a)\right|^{q}+\left[2^{s+1}-1\right]\left|f^{\prime}(b)\right|^{q}}{2^{s}(s+1)}-c \frac{(b-a)^{2}}{6}\right)^{\frac{1}{q}}\right\} .
\end{aligned}
$$


Remark. Choosing $s=1$ in Corollary 2, we obtain the inequality

$$
\begin{aligned}
\left|f\left(\frac{a+b}{2}\right)-\int_{a}^{b} f(x) d x\right| \leq & \frac{b-a}{4}\left(\frac{1}{p+1}\right)^{\frac{1}{p}}\left\{\left(\frac{3\left|f^{\prime}(a)\right|^{q}+\left|f^{\prime}(b)\right|^{q}}{4}-c \frac{(b-a)^{2}}{12}\right)^{\frac{1}{q}}\right. \\
& \left.+\left(\frac{\left|f^{\prime}(a)\right|^{q}+3\left|f^{\prime}(b)\right|^{q}}{4}-c \frac{(b-a)^{2}}{12}\right)^{\frac{1}{q}}\right\} .
\end{aligned}
$$

Corollary 3. If we choose $m=n=\frac{a+b}{2}$ in Theorem 3, then we have

$$
\begin{aligned}
\left|\frac{f(a)+f(b)}{2}-\int_{a}^{b} f(x) d x\right| & \leq \frac{b-a}{2 \times 2^{\frac{1}{p}}}\left(\frac{1}{p+1}\right)^{\frac{1}{p}}\left\{\left(\frac{\left[2^{s+1}-1\right]\left|f^{\prime}(a)\right|^{q}+\left|f^{\prime}(b)\right|^{q}}{2^{s+1}(s+1)}-c \frac{(b-a)^{2}}{12}\right)^{\frac{1}{q}}\right. \\
& \left.+\left(\frac{\left|f^{\prime}(a)\right|^{q}+\left[2^{s+1}-1\right]\left|f^{\prime}(b)\right|^{q}}{2^{s+1}(s+1)}-c \frac{(b-a)^{2}}{12}\right)^{\frac{1}{q}}\right\} .
\end{aligned}
$$

Remark. Choosing $s=1$ in Corollary 3, we obtain the inequality

$$
\begin{aligned}
\left|\frac{f(a)+f(b)}{2}-\int_{a}^{b} f(x) d x\right| & \leq \frac{b-a}{4}\left(\frac{1}{p+1}\right)^{\frac{1}{p}}\left\{\left(\frac{3\left|f^{\prime}(a)\right|^{q}+\left|f^{\prime}(b)\right|^{q}}{4}-c \frac{(b-a)^{2}}{12}\right)^{\frac{1}{q}}\right. \\
& \left.+\left(\frac{\left|f^{\prime}(a)\right|^{q}+3\left|f^{\prime}(b)\right|^{q}}{4}-c \frac{(b-a)^{2}}{12}\right)^{\frac{1}{q}}\right\} .
\end{aligned}
$$

Corollary 4. If we choose $m=\frac{a+5 b}{6}$ and $n=\frac{5 a+b}{6}$ in Theorem 3 , then we have

$$
\begin{aligned}
& \left|\frac{1}{6}\left[f(a)+4 f\left(\frac{a+b}{2}\right)+f(b)\right]-\int_{a}^{b} f(x) d x\right| \leq \frac{b-a}{6}\left(\frac{2^{p+1}+1}{6(p+1)}\right)^{\frac{1}{p}} \\
& \times\left\{\left(\frac{\left[2^{s+1}-1\right]\left|f^{\prime}(a)\right|^{q}+\left|f^{\prime}(b)\right|^{q}}{2^{s+1}(s+1)}-c \frac{(b-a)^{2}}{12}\right)^{\frac{1}{q}}+\left(\frac{\left|f^{\prime}(a)\right|^{q}+\left[2^{s+1}-1\right]\left|f^{\prime}(b)\right|^{q}}{2^{s+1}(s+1)}-c \frac{(b-a)^{2}}{12}\right)^{\frac{1}{q}}\right\} .
\end{aligned}
$$

Remark. Choosing $s=1$ in Corollary 3, we obtain the inequality

$$
\begin{aligned}
\left|\frac{1}{6}\left[f(a)+4 f\left(\frac{a+b}{2}\right)+f(b)\right]-\int_{a}^{b} f(x) d x\right| & \leq \frac{b-a}{12}\left(\frac{2^{p+1}+1}{3(p+1)}\right)^{\frac{1}{p}}\left\{\left(\frac{3\left|f^{\prime}(a)\right|^{q}+\left|f^{\prime}(b)\right|^{q}}{4}-c \frac{(b-a)^{2}}{12}\right)^{\frac{1}{q}}\right. \\
& \left.+\left(\frac{\left|f^{\prime}(a)\right|^{q}+3\left|f^{\prime}(b)\right|^{q}}{4}-c \frac{(b-a)^{2}}{12}\right)^{\frac{1}{q}}\right\} .
\end{aligned}
$$


Theorem 4. Let $f: I \rightarrow \mathbb{R}$ be a differantiable mapping on $I^{0}$ where $a, b \in I$ with $a<b$. If $\left|f^{\prime}\right|$ is strongly s-convex on $[a, b]$ for some $s \in(0,1]$ with modulus $c>0$, then following inequality holds.

$$
\begin{aligned}
& \left|\frac{n}{b-a} \int_{a}^{\frac{a+b}{2}} f(x) d x+\frac{m}{b-a} \int_{\frac{a+b}{2}}^{b} f(x) d x-\frac{n+m}{2} f\left(\frac{a+b}{2}\right)\right| \leq(b-a)\left\{\left[\frac{m}{2^{s+2}(s+2)}+n \frac{2^{s+2}-s-3}{2^{s+2}(s+1)(s+2)}\right]\left|f^{\prime}(a)\right|\right. \\
& \left.+\left[\frac{m}{2^{s+2}(s+2)}+n \frac{2^{s+2}-s-3}{2^{s+2}(s+1)(s+2)}\right]\left|f^{\prime}(b)\right|-\frac{5 c(m+n)}{192}(b-a)^{2}\right\}
\end{aligned}
$$

where $n \in\left[a, \frac{a+b}{2}\right], m \in\left[\frac{a+b}{2}, b\right]$.

Proof. From Lemma 2 and using strongly s-convex of $\left|f^{\prime}\right|$, we have

$$
\begin{aligned}
& \left|\frac{n}{b-a} \int_{a}^{\frac{a+b}{2}} f(x) d x+\frac{m}{b-a} \int_{\frac{a+b}{2}}^{b} f(x) d x-\frac{n+m}{2} f\left(\frac{a+b}{2}\right)\right| \leq(b-a)\left\{\int_{0}^{\frac{1}{2}} m t\left|f^{\prime}((t a)+(1-t) b)\right| d t\right. \\
& \left.+\int_{\frac{1}{2}}^{1} n(1-t)\left|f^{\prime}((t a)+(1-t) b)\right| d t\right\} \leq(b-a)\left\{\int_{0}^{\frac{1}{2}} m t\left[t^{s}\left|f^{\prime}(a)\right|+(1-t)^{s}\left|f^{\prime}(b)\right|-c t(1-t)(b-a)^{2}\right]\right. \\
& \left.+\int_{\frac{1}{2}}^{1} n(1-t)\left[t^{s}\left|f^{\prime}(a)\right|+(1-t)^{s}\left|f^{\prime}(b)\right|-c t(1-t)(b-a)^{2}\right]\right\} \\
& =(b-a)\left\{m\left|f^{\prime}(a)\right| \int_{0}^{\frac{1}{2}} t^{s+1} d t+m\left|f^{\prime}(b)\right| \int_{0}^{\frac{1}{2}} t(1-t)^{s} d t-m c(b-a)^{2} \int_{0}^{\frac{1}{2}} t^{2}(1-t) d t\right. \\
& \left.+n\left|f^{\prime}(a)\right| \int_{\frac{1}{2}}^{1}(1-t) t^{s} d t+n\left|f^{\prime}(b)\right| \int_{\frac{1}{2}}^{1}(1-t)^{s+1} d t-n c(b-a)^{2} \int_{\frac{1}{2}}^{1} t(1-t)^{2} d t\right\}
\end{aligned}
$$

Using the facts that

$$
\begin{aligned}
& \int_{0}^{\frac{1}{2}} t^{s+1} d t=\int_{\frac{1}{2}}^{1}(1-t)^{s+1} d t=\frac{1}{2^{s+2}(s+2)} \\
& \int_{0}^{\frac{1}{2}} t(1-t)^{s} d t=\int_{\frac{1}{2}}^{1}(1-t) t^{s} d t=\frac{2^{s+2}-s-3}{2^{s+2}(s+1)(s+2)}
\end{aligned}
$$

and

$$
\int_{0}^{\frac{1}{2}} t^{2}(1-t) d t=\int_{\frac{1}{2}}^{1} t(1-t)^{2} d t=\frac{5}{192}
$$

one can obtain required result.

Remark. If we choose $m=n$ in Theorem 4 , then Theorem 4 reduces to Remark 2.

Corollary 5. Under assumption of Theorem 4 with $s=1$, we have

$$
\begin{aligned}
\left|\frac{n}{b-a} \int_{a}^{\frac{a+b}{2}} f(x) d x+\frac{m}{b-a} \int_{\frac{a+b}{2}}^{b} f(x) d x-\frac{n+m}{2} f\left(\frac{a+b}{2}\right)\right| \leq & \frac{b-a}{24}\left[(m+2 n)\left|f^{\prime}(a)\right|+(2 m+n)\left|f^{\prime}(b)\right|\right] \\
& -\frac{5 c(m+n)}{192}(b-a)^{3} .
\end{aligned}
$$


Theorem 5. Let $f: I \rightarrow \mathbb{R}$ be a differantiable mapping on $I^{0}$ where $a, b \in I$ with $a<b$. If $\left|f^{\prime}\right|^{q}$ is strongly $s-$ convex on $[a, b]$ for some $s \in(0,1]$ with modulus $c>0$, then following inequality holds :

$$
\begin{aligned}
& \left|\frac{n}{b-a} \int_{a}^{\frac{a+b}{2}} f(x) d x+\frac{m}{b-a} \int_{\frac{a+b}{2}}^{b} f(x) d x-\frac{n+m}{2} f\left(\frac{a+b}{2}\right)\right| \\
& \leq \frac{b-a}{4}\left(\frac{1}{p+1}\right)^{\frac{1}{p}}\left\{m\left(\frac{\left|f^{\prime}(a)\right|^{q}+\left|f^{\prime}(b)\right|^{q}\left[2^{s+1}-1\right]}{2^{s}(s+1)}-\frac{c(b-a)^{2}}{6}\right)^{\frac{1}{q}}\right. \\
& \left.+n\left(\frac{\left|f^{\prime}(a)\right|^{q}\left[2^{s+1}-1\right]+\left|f^{\prime}(b)\right|^{q}}{2^{s}(s+1)}-\frac{c(b-a)^{2}}{6}\right)^{\frac{1}{q}}\right\}
\end{aligned}
$$

where $n \in\left[a, \frac{a+b}{2}\right], m \in\left[\frac{a+b}{2}, b\right]$ and $\frac{1}{p}+\frac{1}{q}=1$.

Proof. From Lemma 2 and using Hölder Inequality, then we have

$$
\begin{aligned}
& \left|\frac{n}{b-a} \int_{a}^{\frac{a+b}{2}} f(x) d x+\frac{m}{b-a} \int_{\frac{a+b}{2}}^{b} f(x) d x-\frac{n+m}{2} f\left(\frac{a+b}{2}\right)\right| \\
& \leq(b-a)\left\{\int_{0}^{\frac{1}{2}} m t\left|f^{\prime}(t a+(1-t) b)\right| d t+\int_{\frac{1}{2}}^{1} n(1-t)\left|f^{\prime}(t a+(1-t) b)\right| d t\right\} \\
& \leq(b-a)\left\{\left(\int_{0}^{\frac{1}{2}}(m t)^{p} d t\right)^{\frac{1}{p}}\left(\int_{0}^{\frac{1}{2}}\left|f^{\prime}(t a+(1-t) b)\right|^{q} d t\right)^{\frac{1}{q}}\right. \\
& \left.+\left(\int_{\frac{1}{2}}^{1} n^{p}(1-t)^{p} d t\right)^{\frac{1}{p}}\left(\int_{\frac{1}{2}}^{1}\left|f^{\prime}(t a+(1-t) b)\right|^{q} d t\right)^{\frac{1}{q}}\right\}
\end{aligned}
$$

Using strongly s-convexity of $\left|f^{\prime}\right|^{q}$;

$$
\left|\frac{n}{b-a} \int_{a}^{l} f(x) d x+\frac{m}{b-a} \int_{l}^{b} f(x) d x-\frac{n+m}{2} f(l)\right| \leq(b-a)\left\{\left(\int_{0}^{\frac{1}{2}}(m t)^{p} d t\right)^{\frac{1}{p}} \theta_{m}+\left(\int_{\frac{1}{2}}^{1}\left[n(1-t)^{p} d t\right]\right)^{\frac{1}{p}} \theta_{m}\right\}
$$

where $\theta_{m}=\left(\int_{\frac{1}{2}}^{1}\left[t^{s}\left|f^{\prime}(a)\right|^{q}+(1-t)^{s}\left|f^{\prime}(b)\right|^{q}-c t(1-t)(b-a)^{2}\right] d t\right)^{\frac{1}{q}}$ and $l=\frac{a+b}{2}$. By simple computation, the desired inequality (26) can be easily established.

Remark. If we choose $m=n$ in Theorem 5, then Theorem 5 reduces to Remark 2.

Corollary 6. Under assumption of Theorem 4 with $s=1$, we have

$$
\left|\frac{n}{b-a} \int_{a}^{\frac{a+b}{2}} f(x) d x+\frac{m}{b-a} \int_{\frac{a+b}{2}}^{b} f(x) d x-\frac{n+m}{2} f\left(\frac{a+b}{2}\right)\right| \leq \frac{b-a}{4}\left(\frac{1}{p+1}\right)^{\frac{1}{p}}\left\{m\left(\theta_{q}\right)^{\frac{1}{q}}+n\left(\theta_{p}\right)^{\frac{1}{q}}\right\},
$$

where $\theta_{p}=\left(\frac{3\left|f^{\prime}(a)\right|^{q}+\left|f^{\prime}(b)\right|^{q}}{4}-\frac{c(b-a)^{2}}{6}\right)$ and $\theta_{q}=\left(\frac{\left|f^{\prime}(a)\right|^{q}+3\left|f^{\prime}(b)\right|^{q}}{4}-\frac{c(b-a)^{2}}{6}\right)$ 


\section{Conclusions}

In this study, we presented some generalized Hermite type inequalities for the mappings whose derivatives are strongly s-convex functions in the second sense are established. A further study could be assess weighted versions of these inequalities.

\section{Competing interests}

The authors declare that they have no competing interests.

\section{Authors' contributions}

All authors have contributed to all parts of the article. All authors read and approved the final manuscript.

\section{References}

[1] H. Angulo, J. Gimenez, A. M. Moros, and K. Nikodem, On strongly h-convex function, Ann. Funct. Anal. 2(2), $2011,85-91$.

[2] M. K. Bakula and J. Pečarić, Note on some Hadamard-type inequalities, Journal of Inequalities in Pure and Applied Mathematics, vol. 5, no. 3, article 74, 2004.

[3] M. V. Cortez, Relative strongly $h$-convex functions and integral inequalities, Appl. Math. Inf. Sci. Lett. 4, No. 2, 1-7 (2016).

[4] S. S. Dragomir and C. E. M. Pearce, Selected Topics on Hermite-Hadamard Inequalities and Applications, RGMIA Monographs, Victoria University, 2000.

[5] S. S. Dragomir and R.P. Agarwal, Two inequalities for differentiable mappings and applications to special means of real numbers and to trapezoidal formula, Appl. Math. lett., 11(5) (1998), 91-95.

[6] H. Hudzik, L. Maligranda, Some remarks on s-convex functions. Aequ. Math. 48, 100-111 (1994).

[7] M. E. Kiris and M. Z. Sarikaya, Some generalized inequalities for Hermite-Hadamard's integral inequalities and applications, submited (2015).

[8] J. Makó and A. Házy, On strongly convex functions. Carpathian Journal of Mathematics, 32 (1). 87-95.

[9] N. Merentes, K. Nikodem, Remarks on strongly convex functions, Aequationes Math. 80 (2010) 193-199.

[10] K. Nikodem, Z. Pales, Characterizations of inner product spaces be strongly convex functions, Banach J. Math. Anal. 5 (2011) $83-87$.

[11] M. E. Özdemir, Ç. Yıldız, A. O. Akdemir and E. Set, On some inequalities for s-convex functions and applications, Journal of Inequalities and Applications 2013, 2013:333.

[12] J.E. Pečarić, F. Proschan and Y.L. Tong, Convex functions, partial orderings and statistical applications, Academic Press, Boston, 1992.

[13] B.T. Polyak, Existence theorems and convergence of minimizing sequences in extremum problems with restictions, Soviet Math. Dokl. 7 (1966), 72-75.

[14] M. Z. Sarikaya, T. Tunc and M. K. Yildiz, Some generalized integral inequalities for convex functions and applications, AIP Conference Proceedings, 1726, 020047 (2016); doi: 10.1063/1.4945873.

[15] M. Z. Sarikaya, On strongly $\varphi_{h}-$ convex functions in inner product spaces, Arabian Journal of Mathematics, (2013) 2:295-302.

[16] M. Z. Sarikaya, E. Set, M. E. Ozdemir and S. S. Dragomir, New some Hadamard's type inequalities for co-ordinated convex functions, Tamsui Oxford Journal of Information and Mathematical Sciences, 28(2) (2012) 137-152. 
[17] M. Z. Sarikaya and H. Yaldiz, On Hermite Hadamard-type inequalities for strongly log-convex functions, International Journal of Modern Mathematical Sciences, 2013, 5(3): 92-98.

[18] M. Z. Sarikaya and H. Yaldiz, On the Hadamard's type inequalities for L-Lipschitzian mapping, Konuralp Journal of Mathematics, 1(2), 33-40 (2013)

[19] M. Z. Sarikaya and K. Ozcelik, On Hermite-Hadamard type integral inequalities for strongly $\varphi_{h}-$ convex functions, International Journal of Advanced Research in Engineering and Applied Sciences (IJAREAS), 1(1), pp:34-52, 2014.

[20] M. Z. Sarikaya, On Hermite Hadamard-type inequalities for strongly $\varphi$-convex functions, Southeast Asian Bull. Math., 39(1) (2015) , pp: 123-132.

[21] M. Z. Sarikaya, E. Set and M. E. Ozdemir, On new inequalities of Simpson's type for s-convex functions, Computers and Mathematics with Applications 60 (2010) 2191-2199. 